\title{
Adipose tatty acid composition and gene expression in obesity, and response to chronic marine omega-3 fatty acid supplementation.
}

\author{
Helena Fisk, Rob Ayres, Caroline Childs, Elizabeth Miles, Rebecca Clarke-Harris, \\ Karen Lillycrop and Philip Calder \\ University of Southampton, Southampton, United Kingdom
}

\begin{abstract}
Introduction: Obesity is an excess of adipose tissue (AT) and is linked with increased inflammation that enhances risk of type-2 diabetes and cardiovascular disease. The BIOCLAIMS study assessed the effect of obesity on AT fatty acid composition and gene expression, and the responses of these to chronic omega-3 FA supplementation.
\end{abstract}

Materials and methods: AT biopsies were collected pre- and post-12 week supplementation with $1.1 \mathrm{~g}$ EPA $+0.8 \mathrm{~g}$ DHA/day or corn oil. The composition of FA in the total lipid extract of AT from 37 normal-weight and 44 obese subjects was assessed by gas chromatography, whole AT transcriptome from 10 normal-weight and 10 obese subjects was assessed by RNA-Sequencing, and selected gene expression in AT of 27 normal-weight and 38 obese subjects was assessed by qRT-PCR.

Results: 789 AT genes were differentially expressed (623 upregulated, 175 downregulated) in obesity compared to normal-weight (FC $>2, P<0.05$ ). Differentially expressed genes included EGFL6, MMP-7 and -9, 5-LOX, WNT3 and WNT10B, DACT2, CNR1, SLC27A2 and PLA2G7, and were associated with immune and inflammatory response, cell proliferation, activation and movement, Wnt signalling, remodelling and expansion, and lipid incorporation and degradation.

Chronic supplementation with EPA + DHA increased the concentration of AT EPA, DPA and DHA in normal-weight subjects $(P$ $<0.01)$, and EPA in obese subjects $(P=0.006)$. EPA + DHA modulated the expression of 26 genes (14 upregulated, 12 downregulated) in normal-weight subjects and 7 genes ( 3 upregulated, 5 downregulated) in obese subjects (FC $>2, P<0.05)$. Of note, EPA + DHA downregulated IGLV1-44, IGLV1-51, PROK2, and TREM1 in normal weight subjects $(P<0.05)$, and IGLV1-44, IGLV1-47, DACT2 and IDO1 obese subjects $(P<0.05)$. Genes of note upregulated by EPA + DHA included KCNH2, GCGR, SLC36A2 and FOXC2 in normal-weight subjects, and MAB21L1, LRRTM4, and COX-2, in obese subjects. Differentially expressed genes were associated with a decrease in complement activation and immunoglobulin secretion, negative regulation of cell proliferation, and positive regulation of remodelling, amino acid and glucose transport, and COX pathway metabolite synthesis.

Discussion: These data indicate an altered AT transcription profile and gene expression in obesity suggesting enhanced immune and inflammatory response, tissue expansion and remodelling, and changes to lipid metabolism, as well as dysregulation in response to supplementary EPA + DHA at a gene expression level. EPA + DHA are able to modulate AT gene expression predominantly associated with reducing immune response, but obesity may involve resistance to the effects on tissue remodelling and nutrient transport.

\section{Conflict of Interest}

There is no conflict of interest 